\title{
Tetanus Antitoxin Titres in Military Personnel
}

Lt Col D N Faithfull-Davies ${ }^{a}$

$M B, M S c$, MRCPath, RAMC

Col E S Parryb

MB, DTM\&H, DRCPath, L/RAMC

Dr F Sheffield

$\mathrm{MB}, \mathrm{ChB}$

Sgt P M Fieldhouse ${ }^{c}$

RAMC

\section{Sgt M A Boxall}

BSc, RAMC

\section{The David Bruce Laboratories, East Everleigh, Marlborough, Wilts, SN8 5HD}

SUMMARY: A study was made of the titres of tetanus antitoxin in the sera of 186 recruits and 886 serving personnel. Allu were found to have titres of more than $0.01 \mathrm{IU}$ per $\mathrm{ml}$, the titre regarded as sufficient to provide protection from tetanus The percentage of recruits with tetanus antitoxin titres $<0.1$ IU per $\mathrm{ml}$ was significantly greater than the percentag $\varphi_{0} g^{5}$ serving personnel with antitoxin titres $<0.1 \mathrm{IU}$ per $\mathrm{ml}$ and the overall geometric mean titre of the antitoxin in the seraDof the recruits was significantly lower than that in the sera of the serving personnel. In the light of the finding that all of the recruits had protective levels of tetanus antitoxin and in view of the imminent withdrawal of TABT Vaccine, alternatife strategies for immunisation against tetanus are presented.

\section{Introduction}

The protection of military personnel against tetanus, especially against tetanus contracted in association with an injury received during training or wounding in combat, is an essential function of the military medical services. Tetanus prophylaxis in the form of three spaced $0.1 \mathrm{ml}$ intradermal doses of TABT vaccine is therefore given to all recruits on entry. Serving personnel are given the same vaccine at three year intervals up to the age of 35 years. After 35 years $0.5 \mathrm{ml}$ doses of tetanus vaccine or tetanus vaccine (adsorbed) are given at five year intervals ${ }^{1}$. Each $0.1 \mathrm{ml}$ dose of TABT vaccine contains $1.8 \mathrm{Lf}$ of tetanus toxoid, each $0.5 \mathrm{ml}$ dose of tetanus vaccine $12 \mathrm{Lf}$ of toxoid and each $0.5 \mathrm{ml}$ dose of tetanus vaccine (adsorbed) not less than $40 \mathrm{IU}$ of immunising potency.

Contrary views of the appropriateness of this immunisation schedule are tenable. On the one hand an immunisation schedule consisting of three doses of diphtheria/tetanus/pertussis vaccine (plain or adsorbegf) in infancy, a reinforcing dose of diphtheria/tetantis vaccine (plain or adsorbed) at school entry, a reinforcige dose of tetanus vaccine (plain or adsorbed) adolescence, and subsequent implementation of theo multidose military schedule may be excessive and leatd to undesirably high titres of tetanus antitoxin in the blood. Such high levels have been associated with the्षि occasional severe local reactions to tetanus vaccine ${ }^{2}$ and it is possible that they may be associated also with rare,, $\overrightarrow{0}$ but alarming, general reactions ${ }^{3}$. On the other hand, the 3 small amount of tetanus toxoid (1.8 LF) in the $0.1 \mathrm{mP}$ dose of TABT vaccine may be insufficient to stimulate a? "secondary" immune response and thus, despite regular administration, the titres of antitoxin induced by immunisation prior to entry into the services may fall to levels which no longer provide protection. A further $\overrightarrow{\bar{\sigma}}$ complication is that it is possible that a small number of recruits may, for various reasons, enter the services

${ }^{a}$ Now at The Leishman Laboratory, Cambridge Military Hospital, Aldershot, Hants, GU11 $2 A N$

${ }^{b}$ Now at The Chemical Defence Establishment, Porton Down, Salisbury, Wilts, SP4 OJQ

'Now Mr P M Fieldhouse, clo A V Howe Company Ltd, 12-14 St Anne Crescent, London SWI8 2LS

${ }^{d}$ Now at The Queen Elizabeth Military Hospital, Stadium Road, Woolwich, London SE184QH

'The David Bruce Laboratories closed in October 1988 
without having received any form of prior immunisation $^{4,5}$.

To obtain information on the effects of the military immunisation schedule on the personnel to whom it is applied, and thus an indication of the extent to which the schedule provides immunity to tetanus, blood samples were obtained from recruits prior to the first immunisation in their military careers and from immunised serving personnel between the ages of 18 and 40 years. This paper reports the titres of tetanus antitoxin found in the sera from the recruits and the serving personnel and, in the light of the findings and the imminent withdrawal of TABT vaccine, presents alternative strategies to the current military schedule.

\section{Materials and Methods}

Ethical approval for the study was sought and obtained from the Army Medical Research Executive.

\section{Volunteer donors}

Blood for examination was obtained from volunteer military donors at blood donation sessions conducted by the staff of the Army Blood Supply Depot. Each donor was told of the purpose of the study and was asked to provide a $10 \mathrm{ml}$ sample of blood at the end of the donation. The donor was also asked to permit a Royal Army Medical Corps clerk to examine his medical record envelope (F Med 4) and to copy from it on to record sheets the relevant details of the immunisation history. Those who agreed were further required to sign a consent sheet to signify their willingness to participate in the study.

\section{Treatment of blood samples and estimation of antitoxin}

The blood samples were taken into dry tubes and allowed to clot. The clots were loosened with a sterile pasteur pipette and the clotted samples were centrifuged. The serum was drawn off, placed in plastic tubes and maintained in the frozen state $\left(-20^{\circ} \mathrm{C}\right)$ until needed for assay.

The titres of tetanus antitoxin in the serum samples obtained from the donors were estimated by two in vitro methods. The first of these was a commercially available enzyme immunosorbent assay (EIA) kit (Labsystems (UK) Ltd, The Ringway Centre, Basingstoke, UK) and a Titertek Multiscan microtitre plate reader (Titertek, UK) and the second the parallel line micro-ELISA procedure (ELISA) described by Melville-Smith et at . In the second method the British reference preparation for Human Tetanus Immunoglobulin was used as the reference material and the optical densities of the reaction mixtures were read with a Titertek Multiscan microplate reader. The $\log$ dose-log response lines obtained with each serum were drawn manually and the antitoxin titres were calculated from the horizontal distance between the log dose-log response line of each test preparation and that of the reference preparation.
The antitoxin titres of selected sera were, additionally, estimated by neutralisation tests in mice (MNT) by the method described in the monograph for tetanus antitoxin in the British Pharmacopoeia $1988^{7}$ with two modifications. Firstly, in order to increase the sensitivity of the assay to $0.01 \mathrm{IU}$ of antitoxin per $\mathrm{ml}$, the amount of toxin used was reduced to the $L p / 400$ dose and, secondly, the serial dilutions of the sera were made in 1.6 fold steps. The British Standard for Tetanus Antitoxin was used as the reference preparation.

\section{Results}

Blood samples were obtained from 1072 individuals of whom 186 were recruits, 825 serving personnel up to and including 35 years old and 61 serving personnel more than 35 years old. All of the sera were assayed for tetanus antitoxin by the Labsystems procedure and the distributions of the titres in the sera from the three groups are shown in the histograms in Figure 1. Overall 1063 of the 1072 sera $(99 \%)$ were found to have titres $>0.1$ International Unit (IU) of tetanus antitoxin per $\mathrm{ml}$, this titre being ten times that generally accepted as sufficient to provide immunity to tetanus ${ }^{8}$. Seven of the nine sera with titres $<0.1 \mathrm{IU}$ per $\mathrm{ml}$ were from recruit and there was one from each of the other two groups:This difference in the proportions of recruits (7/186) and serving soldiers $(2 / 886)$ with titres $<0.1$ IU per $\mathrm{ml}$ wa found by Fisher's exact probability test ${ }^{9}$ to be highl significant $(\mathrm{p}<0.005)$.

The geometric mean titre of the sera from the recruits was $1.09 \mathrm{IU}$ per $\mathrm{ml}$ and that of the sera from the serving personnel $1.47 \mathrm{IU}$ per $\mathrm{ml}$, the difference bein significant $(0.002<\mathrm{p}<0.01)$. Subdivision of the ser from the serving personnel into a subset from personnet up to and including 35 years of age and a subset from personnel over 35 years of age revealed that the mean antitoxin titre in the younger group was $1.51 \mathrm{IU}$ per $\mathrm{ml}$ and that in the older group $1.12 \mathrm{IU}$ per $\mathrm{ml}$, this difference also being significant $(0.02<\mathrm{p}<0.05)$. Subsequent examination of the records of the serving personnel showed that in the younger group 86 per cent had been immunised in the three years prior to the taking of the blood sample and the mean interval between the last dose of vaccine and the taking of the sample was 581 days; in the older group only 59 per cent had been immunised in the three years prior to the taking of the sample and the mean interval between the last dose and the taking of the sample was 1003 days.

Although the Labsystems EIA has the advantage that it makes possible the assay of large numbers of sera very economically it does not provide evidence that each analyte behaves as if it were a dilution of the reference preparation with which it is compared - a necessary condition for a truly valid bioassay. The sera from 170 of the recruits were therefore reassayed by a parallel line micro-ELISA similar to that of Melville-Smith et $\mathrm{al}^{6}$. The linearity and parallelism of the log dose-log 


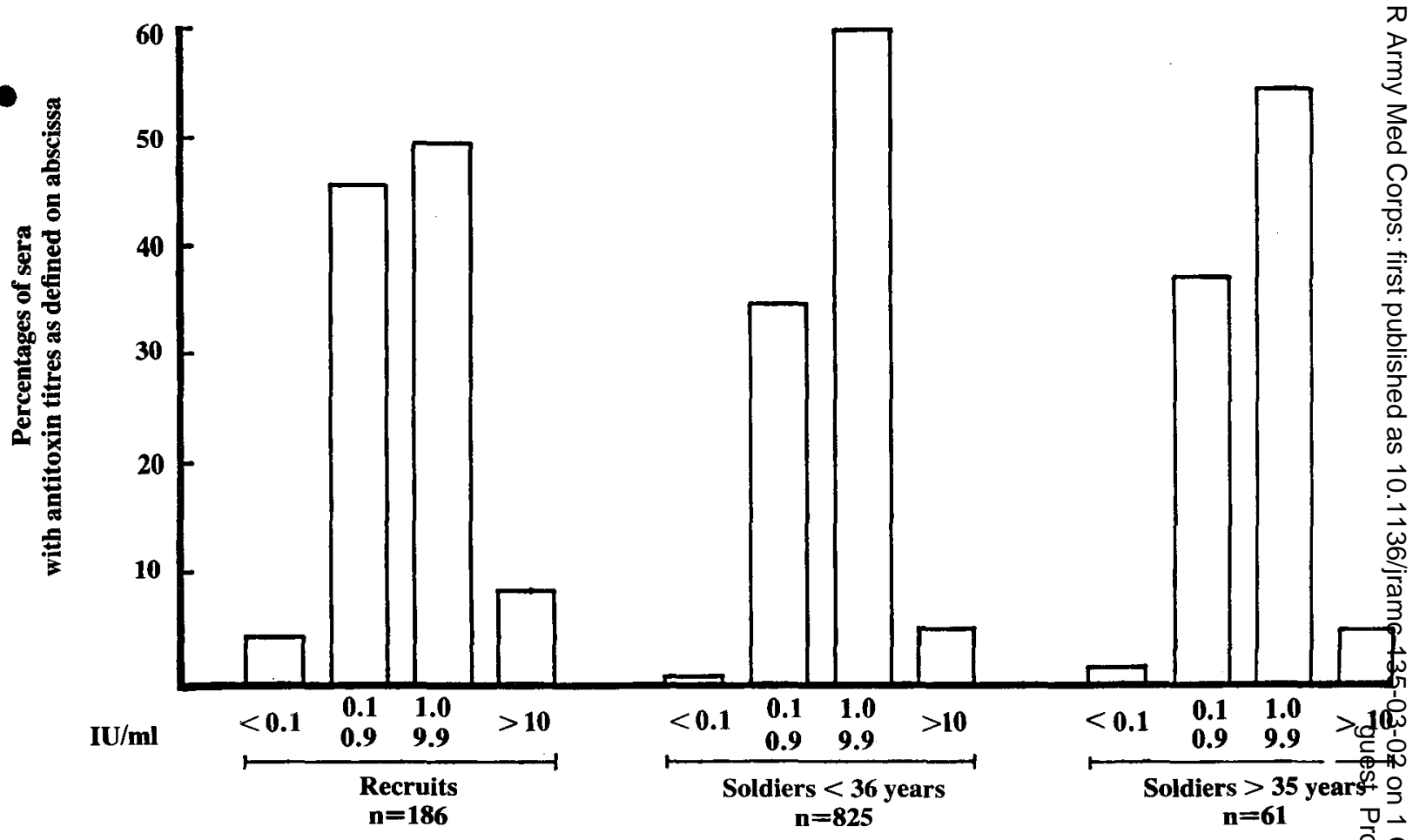

Fig 1. Histograms showing the distributions of the tetanus antitoxin titres of the sera of recruits and serving soldiers和禺 to and including 35 years of age and over 35 years of age.

response relationships were good but overall the correlation between the results obtained with the Labsystems EIA and the micro-ELISA was only fair $(\mathrm{r}=0.82)$. Of the seven sera from recruits found by EIA to have titres $<0.1 \mathrm{IU}$ per $\mathrm{ml}$ six were found by microELISA to have titres in the range 0.07 to 0.12 IU per $\mathrm{ml}$ but the seventh was found to have a titre of $2.3 \mathrm{IU}$ per $\mathrm{ml}$. Furthermore ten sera that were found to have titres $>0.1$ IU per $\mathrm{ml}$ by EIA yielded titres of $<0.1$ IU per $\mathrm{ml}$ by Micro-ELISA, the values ranging from 0.04 to 0.09 IU per ml. Sera from 377 of the serving soldiers were tested also by micro-ELISA and none was found to have a titre $<0.1$ IU per $\mathrm{ml}$.

In view of the report by Simonsen $e t a l^{10}$ that titres $<0.16$ IU per $\mathrm{ml}$ obtained by enzyme linked immunoassays of tetanus antitoxin may overestimate the antitoxin level by reason of non-specific binding, 15 sera obtained from the recruits were titrated for antitoxin by neutralisation tests in mice. The sera were those from among the recruits' samples that had the lowest in vitro titres and were available in sufficient quantities for neutralisation tests. The results obtained with these 15 sera in the three different assay systems, EIA, ELISA and MNT, are given in Table 1 which shows that the MNT yielded results that were higher
Table 1

Titres of tetanus antitoxin in the sera of 15 recruits priotồio immunisation with TABT vaccine. The columns show the tit:? obtained by each of three assay methods; neutralisation test in mice (MNT), the Labsystems immunoassay (EIA) and tho parallel line micro-ELISA (ELISA)

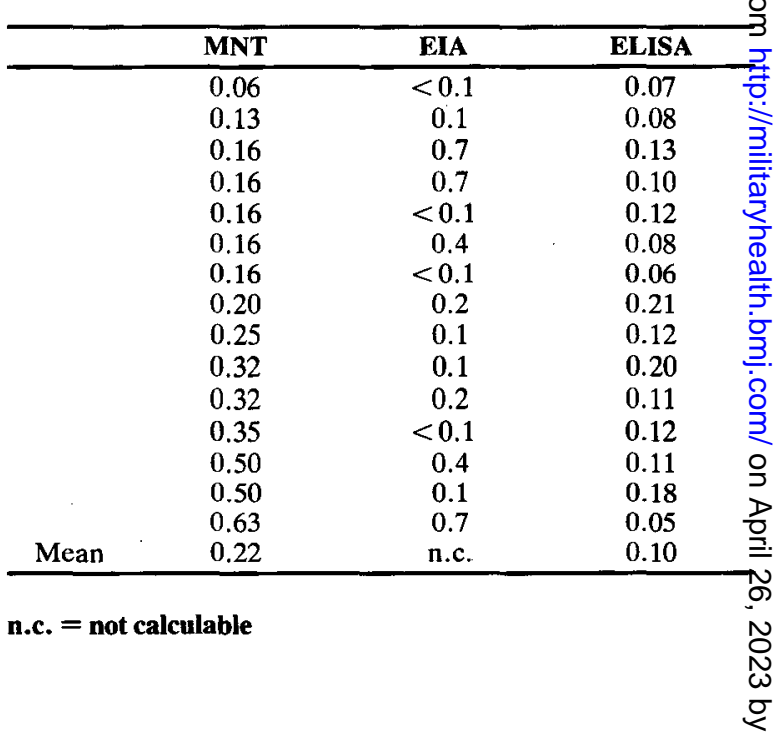


than those provided by the EIA in ten cases and higher than those provided by the micro-ELISA in 13.

The titres obtained by EIA were analysed in relation to the numbers of doses of vaccine that had been given and the number of days that had elapsed between the last dose of vaccine and the taking of the blood sample but no correlation either positive or negative was found in either case.

\section{Discussion}

The efficacy of tetanus toxoid in the prophylaxis of tetanus was convincingly demonstrated in the Second World War by the low incidence of tetanus in the armed forces of the Allies and the high incidence in the unimmunised land forces of the Axis powers ${ }^{11}$. There remains, however, the problem of ensuring that protection is maintained by the use of adequate reinforcing doses of vaccine while, at the same time, avoiding overdosage which can lead to unnecessarily high titres, unduly severe reactions ${ }^{2}$, and waste of vaccine and stores.

The titre of antitoxin which is generally accepted as sufficient to provide protection against tetanus is $0.01 \mathrm{IU}$ per $\mathrm{ml}^{8}$. The findings of this study thus show that all personnel who entered the study were protected. This degree of protection attests to the efficacy of the national immunisation programme but it does not necessarily follow that all recruits have protective levels of antitoxin. Indeed, the method of Klopper and Pearson ${ }^{12}$ predicts that, if in a sample of 186 as described in this paper all are protected, as many as 1 per cent may be unprotected in the global population. In the case of a sample of 5,000 , all of whom are protected, the method predicts that not more than 0.2 per cent of the global population may be unprotected.

The findings that sera with low titres of antitoxin, that is titres $<0.1 \mathrm{IU}$ per $\mathrm{ml}$, were significantly more common among the sera from the recruits (7/186) than among the sera from the serving personnel $(2 / 886)$ and that the mean antitoxin titre of the sera from the recruits $(1.06$ IU per $\mathrm{ml}$ ) was significantly lower than that of the serving personnel (1.47 IU per $\mathrm{ml}$ ), suggest that the small amount of tetanus toxoid in TABT vaccine is sufficient to induce a "secondary" response. However, the simultaneous finding that sera with high titres of antitoxin, that is titres $>10 \mathrm{IU}$ per $\mathrm{ml}$, were more common in the sera of recruits $(16 / 186)$ than in the sera of serving personnel (44/886) tends to refute any suggestion that multiple doses of TABT vaccine raise antitoxin titres to levels likely to be associated with adverse reactions. The immunisation schedule with TABT thus appears to serve the useful purpose of maintaining antitoxin titres above the very satisfactory level of 0.1 IU per $\mathrm{ml}$ without inducing levels that might be associated with undesirable side-effects.

The significant difference between the geometric mean titre of antitoxin in the sera of the serving personnel up to and including 35 years of age (1.51 IU per $\mathrm{ml}$ ) and that of the sera of serving personnel over 35 years of age (1.12 IU per $\mathrm{ml}$ ) is possibly related to the longer interval between reinforcing doses in the two groups, this interval being three years in the younger group and five in the older. Support for this view is provided by the markedly longer interval between the last dose of vaccine and the date of the taking of the blood sample in the older group and the smaller percentage of individuals in the group who had received the last dose of vaccine within the three year period prior to the sampling.

Overall, the findings of this study show that the combined effects of the national immunisation programme as defined in "Immunisation against Infectious Disease"13 and the military immunisation programme as defined in "The Memorandum on Immunological Procedures" 1 provide service personnel with excellent immunity to tetanus. It is reasonable to suppose that the findings are, at least in part, reflections of the three doses of TABT vaccine which are given routinely to each recruit and the reinforcing doses that are given to serving personnel. Thus even in a recruit with no previous exposure to tetanus toxoid, the three spaced doses of toxoid in TABT vaccine, small thougho they are $(1.8 \mathrm{Lf})$, may be sufficient to raise a protective्ठ titre of tetanus antitoxin. When, as is imminent, TABT + vaccine is withdrawn and immunisation against tetanus is achieved with tetanus vaccine or tetanus vaccine (adsorbed), the single stimulus of tetanus toxoid, albeip a larger one, may in an individual without previous exposure to the antigen merely prime the immuneo system instead of inducing a protective antitoxin titre The possibility that up to 1 per cent of recruits may not? have protective antitoxin titres on entry or even after single dose of vaccine thus deserves attention. At the same time it is necessary to appreciate that the antigenic content of both plain and adsorbed tetanus vaccine is approximately seven times that of TABT vaccine and that overimmunisation may lead to severe and undesirable side effects and even to adverse reactions. Five strategies to achieve the prophylaxis of tetanus are outlined:

1. Administration to all recruits of three doses of tetanus vaccine (adsorbed) with a six to twelve week interval between the first and second doses and a six to twelve month interval between the second and third doses. This policy would certainly ensure the protection of all recruits but it would be difficult on ethical grounds to justify the administration of three doses to recruits likely to have protective levels even before entry. Furthermore the induction of high titres by the first and second doses might lead to severe side effects, even adverse reactions, after the subsequent doses. Such side effects would be clearly attributable to the tetanus toxoid and would not be obscured by reactions to the salmonella component of the TABT vaccine.

2. Administration to all recruits of one dose of tetanus vaccine (adsorbed). Recruits with previous experience 
of tetanus toxoid would have their antitoxin titres increased and those without previous experience of the toxoid would be immunologically primed. The latter may be expected to respond to a routine reinforcing dose of tetanus vaccine (adsorbed) or a dose given after injury or wounding but post-exposure prophylaxis may, in such circumstances, be complicated by a need for human tetanus immunoglobulin ${ }^{13,14,15}$.

3. Extension of the study to the assay of the sera from a sufficient number of recruits to show that so few are unprotected on entry that their identification is unrealistic. Such an extension might be conducted by EIA at the Army Blood Supply Depot (ABSD) on samples of blood obtained from recruits at the time of venepuncture for blood grouping. Although the results reported in this paper are not in agreement with the findings obtained by Simonsen et $a^{10}$ that sera with low titres of tetanus antitoxin as assayed by the MNT yield spuriously high titres when assayed by micro-ELISA, it would be desirable to confirm all titres below 0.16 IU per $\mathrm{ml}$ by assay in MNTs.

4. Routine immunoassay of sera from all recruits and immunisation in accordance with the titres found. This also could be achieved by EIA at the ABSD. As in (3) above it would be desirable, at least for a time, to confirm low titres obtained by immunoassay by MNTs. This strategy might be refined to provide those recruits without measurable antitoxin with three doses of tetanus vaccine (adsorbed), those with measurable levels up to 0.2 IU per $\mathrm{ml}$ with one dose of tetanus vaccine (adsorbed) and to postpone reinforcement of those with titres greater than 0.2 IU per $\mathrm{ml}$.

5. The acquisition from the germane health authority of each recruit's immunisation record and subsequent immunisation in accordance with the perceived need. As many health authorities now hold immunisation records in data banks the information should be readily available to the local medical officer who undertakes the recruit's pre-entry medical examination. There would be a need to transfer the information to a recruit's training centre where the medical officer would give tetanus vaccine as required. Additionally it would be necessary to provide unit medical officers with protocols defining when vaccine should be given and when it should be witheld.

In the case of each strategy subsequent doses of tetanus vaccine would be given at ten year intervals and after injury in accordance with the "Memorandum on Immunological Procedures ${ }^{1}$ and "Immunisation against infectious disease ${ }^{12}$.

In view of the long term financial implications of immunisation against tetanus, the authors favour option 3. Confirmation of the findings of this study in the larger study would be adequate justification for immunisation of recruits with a single dose of vaccine only. Alternatively, a finding that more than 0.2 per cent of recruits had titres lower than the protective level would provide justification for the implementation of option 4 . Option 5 will become practicable only when all recruits' immunisation records are available on a computer network.

It is important to appreciate that all of the findings reported in this paper have been obtained from recruits to the Regular Army and from serving personnel in the Regular Army. They cannot be extrapolated with 응 confidence to recruits of the Territorial Army Volunteer of Reserve (TAVR), who tend to be older than recruits to the Regular Army, or to TAVR personnel, who may be subjected to less structured immunisation. Before a $\bar{C}$ meaningful policy decision could be taken in respect of $\underline{\bar{\sigma}}$ tetanus immunisation in the TAVR a study similar to that described in this paper would be needed.

\section{Acknowledgements}

The authors are pleased to recognise the contributions $\vec{\omega}$ of Maj Gen (Retd) J C Crook and the donor team of the Army Blood Supply Depot who obtained the blood samples and to Capt D Gault, RAPC (formerly RAMC) for organising the enthusiasm of the clerical staff and the $\vec{\omega}$ combat medical technicians of the David Bruce or Laboratories.

The work reported in this paper was supported by the Army Medical Research Executive under project grate number 320 .

\section{REFERENCES}

1. Memorandum on Immunological Procedures. Joi Services Publication 311. London, Her Majesty's Stationery Office, 1968 as amended.

2. Collier L H, Polokoff S and Mortimer J. Reactions ami antibody responses to reinforcing doses of plain a adsorbed tetanus vaccines. Lancet 1979; i: 1364-1368.

3. Brindle $M J$ and Twyman D G. Allergic reactions to tetanus toxoid. $B r$ Med J 1962; i: 1117-1117.

4. Bainton D. et al. Immunity of children to diphtheria, tetanus and poliomyelitis. Br Med J 1979; i: 854-857.

5. JONES E A, et al, Adverse reactions in adolescents to reinforcing doses of plain and adsorbed tetanus vaccines. Community Medicine 1985; 7: 99-106.

6. Melville-Smith M E, S EAGroatt V A and Watkins J T. A comparison of enzyme linked immunosorbent assay (ELISA) with the toxin neutralisation test in mice as a method for the estimation of tetanus antitoxin in human sera. J Biol Stand 1983; 11: 137-144.

7. British Pharmacopocia 1988 Volume II; A156b-157a. London, Her Majesty's Stationery Office, 1988.

8. TASMAN $A$ and HUYGEN $F \mathbf{J}$ A. Immunisation against tetanus of patients given injections of antitetanus serum. Bull WHO 1962; 26: 397-407.

9. FISHER R A. Statistical methods for research workers 5 th $\frac{\circ}{3}$ Ed. Edinburgh, Oliver and Boyd, 1934.

10. Simonsen $O, B$ Bentzon M W and Heron I. ElisA for the $\frac{O}{J}$ routine determination of antitoxic immunity to tetanus. $J$ I Biol Stand 1986; 14: 231-239.

11. Topley and Wilson's Principles and Practice of Bacteriology, Virology and Immunity. Volume $3 ; 359 \mathrm{a}$. $\Omega$ London, Edward Arnold, 1983/4. 
12. Clopper C J and Pearson E S. Use of confidence or fiducial limits illustrated in the case of the binomial theorem. Biometrika 1934 ; 36: 404-413.

13. Immunisation against Infectious Disease. London, Her Majesty's Stationery Office, 1988.
14. Smith J W G, Lawrence D R and Evans D G. Prevention of tetanus in the wounded. Br Med J 1975; 3: 453-455.

15. ShefField F. To give or not to give. Community View 1985 33: 8-9.

\section{ACADEMIC ACHIEVEMENTS}

FFCM

FRCR

FRCS (Ophthalmology)

MRCPsych

FC Anaes

MFOM

DCH

DTM\&H

DIMC (RCSE)
Brig D H Coombe, L/RAMC

Major CJ Phillips, RAMC

Captain J G F Dowler, RAMC

Major A Gillham RAMC

Captain B C Connell, RAMC

Major R J Claydon, RAMC

Major A K L Niemiro, RAMC

Major T P Finnegan, RAMC

Major M G Braithwaite, RAMC

Captain J P North, RAMC

Major C J R Parker, RAMC

Captain M J Farrar, RAMC 